\title{
Berberine sensitizes TRAIL-induced apoptosis through proteasome-mediated downregulation of c-FLIP and Mcl-1 proteins
}

\author{
SUNG-JUN LEE ${ }^{1}$, HYO-JEONG NOH ${ }^{1}$, EON-GI SUNG ${ }^{1}$, IN-HWAN SONG ${ }^{1}$, \\ JOO-YOUNG KIM ${ }^{1}$, TAEG KYU KWON ${ }^{2}$ and TAE-JIN LEE ${ }^{1}$ \\ ${ }^{1}$ Department of Anatomy, College of Medicine, Yeungnam University, 317-1 Daemyung-Dong Nam-Gu, \\ Daegu 705-717; ${ }^{2}$ Department of Immunology, School of Medicine, Keimyung University, \\ 2800 Dalgubeoldaero, Dalseo-Gu, Daegu 704-701, Republic of Korea
}

Received September 21, 2010; Accepted November 29, 2010

DOI: $10.3892 /$ ijo.2010.878

\begin{abstract}
Berberine (BBR) is an isoquinoline alkaloid which has a wide spectrum of clinical applications including antitumor, anti-microbial and anti-inflammatory activities. In this study, we showed that co-treatment with subtoxic doses of BBR and tumor necrosis factor-related apoptosis-inducing ligand (TRAIL) induced apoptosis in human renal cancer cells, Caki cells, but not in normal tubular kidney cells. Treatment of Caki cells with BBR resulted in downregulation of c-FLIP and Mcl-1 proteins in a dose-dependent manner. The BBR-induced downregulation of c-FLIP and Mcl-1 proteins were involved in proteasome dependent pathways, which was confirmed by the result that pre-treatment with the proteasome inhibitor MG132 inhibited berberine-induced downregulation of the c-FLIP and Mcl-1 proteins. Pretreatment with $\mathrm{N}$-acetyl-1-cysteine (NAC) significantly inhibited the cell death induced by the combined treatment with BBR and TRAIL as well as recovered the expression levels of c-FLIP and Mcl-1 downregulated by treatment with BBR. These results suggested that BBR-stimulated TRAILinduced apoptosis is dependent on the generation of reactive oxygen species through the downregulation of c-FLIP and Mcl-1 proteins. In conclusion, this study demonstrates that BBR enhances TRAIL-induced apoptosis in human renal cancer cells by ROS-mediated c-FLIP and Mcl-1 downregulation.
\end{abstract}

\section{Introduction}

Tumor necrosis factor (TNF)-related apoptosis-inducing ligand (TRAIL) is a potential new anticancer drug. TRAIL

Correspondence to: Dr Tae-Jin Lee, Department of Anatomy, College of Medicine, Yeungnam University, 317-1 DaemyungDong Nam-Gu, Daegu 705-717, Republic of Korea

E-mail: tjlee@med.yu.ac.kr

Key words: berberine, TRAIL, c-FLIP, Mcl-1, ROS, proteasome induces apoptosis in various types of cancer cells in vitro and in vivo, but has little or no toxicity against normal cells, which is supported by the presence of large numbers of decoy receptors on normal cells $(1,2)$. A soluble recombinant TRAIL is undergoing a phase I clinical trial for the treatment of solid tumors (3). However, recent studies have shown that some cancer cells are resistant to the apoptotic effects of TRAIL (4-6), indicating that the single treatment with TRAIL may be insufficient for cancer therapy. Some studies demonstrated that several dietary compounds including resveratrol (6), withaferin A (7), and curcumin (8) sensitize different cancer cells to TRAIL-mediated apoptosis.

Berberine (BBR), a natural alkaloid, is isolated from many medicinal herbs, such as the Chinese herb Huanglian, berberis aquifolium, and berberis vulgaris (9). It has been shown to exhibit multiple pharmacological activities such as inhibition of DNA synthesis, G1 cell cycle arrest, and anticancer effect $(10,11)$. BBR has been reported to inhibit expression of cyclooxygenase-2 (COX-2), to exert anti-metastatic properties in non-small lung cancer cells, as well as to induce apoptosis via promoting the expression of caspase, apoptosisinducing factor (AIF) and endonuclease $\mathrm{G}$ in human tongue squamous carcinoma cancer cells (12). In addition, BBR also sensitizes human glioma cells to ionizing radiation in vitro (13). It was also reported that co-administration with BBR and doxorubicin in xenografted human tongue squamous carcinoma cancer reduced tumor growth at the concentration of doxorubicin without any loss of body weight (14).

Although a large number of studies also showed that BBR possesses anti-tumor activity, the anti-cancer effect of the combination of BBR and TRAIL is not clear. In the present study, we examined the therapeutic potential of BBR on TRAIL-mediated apoptosis and the potential application of $\mathrm{BBR}$ as a complimentary therapeutic agent for human renal cancer cells.

\section{Materials and methods}

Cells and materials. Caki cells were obtained from the American Type Culture Collection (ATCC, Rockville, MD). 
Primary culture of human mesangial cells (Cryo NHMC) and its corresponding growth medium (CC-3146 MsGM) were purchased from Clonetics (San Diego, CA). The culture medium used throughout these experiments was Dulbecco's modified Eagle's medium (DMEM), containing 10\% fetal calf serum (FCS), $20 \mathrm{mM}$ HEPES buffer and $100 \mu \mathrm{g} / \mathrm{ml}$ gentamicin. BBR was directly added to cell cultures at the indicated concentrations. Anti-Bcl-2, anti-PARP, antiprocaspase-3, anti-DR5, anti-Mcl-1, and anti-actin antibodies were purchased from Santa Cruz Biotechnology Inc. (Santa Cruz, CA, USA). Anti-c-FLIP antibody was purchased from Alexis Corporation (San Diego, CA). Berberine was obtained from Sigma Chemical Co. Recombinant human TRAIL/Apo2 ligand (the nontagged $19 \mathrm{kDa}$ protein, amino acid 114-281) was purchased from KOMA Biotech Inc. (Seoul, Korea).

Western blotting. Cellular lysates were prepared by suspending $1 \times 10^{6}$ cells in $100 \mu \mathrm{l}$ of lysis buffer $(137 \mathrm{mM} \mathrm{NaCl}, 15 \mathrm{mM}$ EGTA, $0.1 \mathrm{mM}$ sodium orthovanadate, $15 \mathrm{mM} \mathrm{MgCl}_{2}, 0.1 \%$ Triton X-100, $25 \mathrm{mM}$ MOPS, $100 \mu \mathrm{M}$ phenylmethylsulfonyl fluoride, and $20 \mu \mathrm{M}$ leupeptin, adjusted to $\mathrm{pH}$ 7.2). The cells were disrupted by sonication and extracted at $4^{\circ} \mathrm{C}$ for $30 \mathrm{~min}$. The proteins were electrotransferred to Immobilon-P membranes (Millipore Corporation, Bedford, MA, USA). Detection of specific proteins was carried out with an ECL Western blotting kit according to the manufacturer's instructions.

Cell count and flow cytometry analysis. Cell counts were performed using a hemocytometer. Approximately $1 \times 10^{6}$ Caki cells were suspended in $100 \mu \mathrm{l}$ of PBS, and $200 \mu \mathrm{l}$ of $95 \%$ ethanol were added while vortexing. The cells were incubated at $4^{\circ} \mathrm{C}$ for $1 \mathrm{~h}$, washed with PBS, and resuspended in $250 \mu \mathrm{l}$ of $1.12 \%$ sodium citrate buffer ( $\mathrm{pH} 8.4$ ) together with $12.5 \mu \mathrm{g}$ of RNase. Incubation was continued at $37^{\circ} \mathrm{C}$ for $30 \mathrm{~min}$. The cellular DNA was then stained by applying $250 \mu \mathrm{l}$ of propidium iodide $(50 \mu \mathrm{g} / \mathrm{ml})$ for $30 \mathrm{~min}$ at room temperature. The stained cells were analyzed by fluorescent activated cell sorting (FACS) on a FACScan flow cytometer for relative DNA content based on red fluorescence.

DAPI staining. The cells were fixed with $1 \%$ paraformaldehyde on slide glass for $30 \mathrm{~min}$ at room temperature. After washing with PBS, 300 nM DAPI (40-60-diamidino-2phenylindole, Roche, Germany) was added to the fixed cells for $5 \mathrm{~min}$, after which they were examined by fluorescence microscopy. Apoptotic cells were identified by condensation and fragmentation of nuclei.

Measurement of reactive oxygen species. The intracellular accumulation of ROS was determined using the fluorescent probes $2^{\prime}, 7^{\prime}$-dichlorodihydrofluorescein diacetate ( $\left.\mathrm{H}_{2} \mathrm{DCFDA}\right)$. Briefly, Caki cells were incubated with combination of BBR for $24 \mathrm{~h}$. The cells were stained with $10 \mu \mathrm{M} \mathrm{H}_{2}$ DCFDA for $40 \mathrm{~min}$ at $37^{\circ} \mathrm{C}$ and then observed under a fluorescence microscope (Axiovert 200M, Carl Zeiss). Caki cells were incubated with combination of BBR and TRAIL for $24 \mathrm{~h}$ and loaded with $5 \mu \mathrm{M} \mathrm{H}_{2}$ DCFDA for $1 \mathrm{~h}$ before harvesting. The fluorescence was measured at the desired time intervals by flow cytometry. The ROS generation was assessed by the dichlorofluorescein fluorescence intensity (FL-1, $530 \mathrm{~nm}$ ) from 10,000 cells with a FACScan flow cytometer (BectonDickinson).

RNA isolation and reverse transcriptase-polymerase chain reaction $(R T-P C R)$. To determine whether the potential sensitizing effects of BBR to TRAIL-mediated apoptosis were a result of increased levels of mRNA encoding DR5, we compared the levels of c-FLIP $\mathrm{L}_{\mathrm{L}}$ and Mcl-1 in Caki cells, which were treated with or without various concentrations of BBR. The expression of c-FLIP ${ }_{\mathrm{L}}$ and Mcl-1 was determined by RT-PCR. Total cellular RNA was extracted from cells using the TRIzol reagent (Life Technologies). A cDNA was synthesized from $2 \mu \mathrm{g}$ of total RNA using M-MLV reverse transcriptase (Gibco-BRL, Gaithersburg, MD). The cDNA for c-FLIP $\mathrm{L}_{\mathrm{L}}, \mathrm{Mcl}-1$ and actin were amplified by PCR with specific primers. The sequences of the sense for $c-$ FLIP $_{L}$ were 5'-CGGACTATAGAGTGCTGATGG-3' and the antisense primer for 5'-GATTATCAGGCAGATTCCTAG-3' (c-FLIP ${ }_{\mathrm{L}}$ ), respectively. PCR products were analyzed by agarose gel electrophoresis and visualized by ethidium bromide.

Statistical analysis. Three or more separate experiments were performed. Statistical analysis was done by paired Student's t-test or ANOVA. A p $<0.05$ was considered to have pronounced difference between experimental and control groups.

\section{Results}

BBR sensitizes renal cancer cells to TRAIL-mediated apoptosis. In an attempt to search for novel strategies to overcome TRAIL resistance in cancer cells, we investigated the effect of the combined treatment with BBR and TRAIL in human renal cancer cell line, Caki cell. Co-treatment of Caki cells with BBR and TRAIL resulted in a markedly increased accumulation of sub-G1 phase cells, compared with Caki cells treated with BBR or TRAIL alone (Fig. 1A). In addition, the combined treatment of Caki cells with BBR and TRAIL strongly led to a reduction of the protein levels of $32-\mathrm{kDa}$ precursor together with a concomitant cleavage of PARP, a substrate protein of caspases (Fig. 1B). Next, we analyzed nuclear condensation, which is another hallmark of apoptosis. Combinational treatment with BBR plus TRAIL induced the nuclear condensation in Caki cells. In contrast, nuclear condensation in Caki cells treated with TRAIL alone or BBR alone was barely detected (Fig. 1C). To examine whether cotreatment with BBR and TRAIL affected renal cancer ACHN cells, we investigated the effect of the combined treatment with BBR and TRAIL in ACHN cell. Co-treatment of ACHN cells with BBR and TRAIL resulted in a markedly increased accumulation of sub-G1 phase cells, compared with Caki cells treated with BBR or TRAIL alone (Fig. 1D). Next, we investigated whether co-treatment with BBR and TRAIL affected TCMK-1 normal renal tubular epithelial cell. Interestingly, TCMK-1 cells were resistant to $60 \mu \mathrm{M}$ $\mathrm{BBR}$ or $100 \mathrm{ng} / \mathrm{ml}$ TRAIL alone, and cell morphologies were not significantly affected by treatment with a combination of $60 \mu \mathrm{M}$ BBR and $100 \mathrm{ng} / \mathrm{ml}$ TRAIL (Fig. 1E). 
A

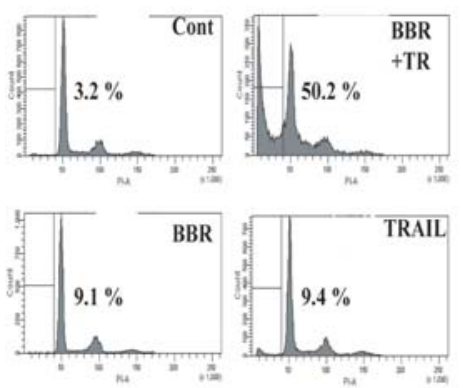

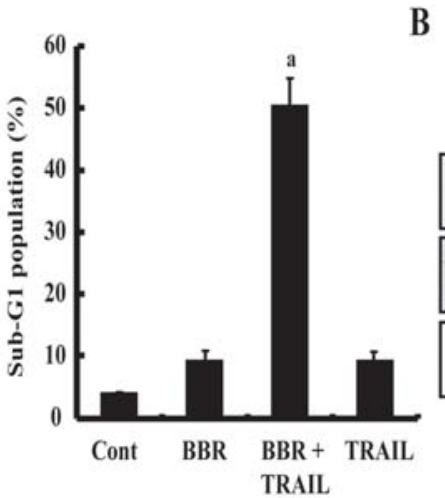

D

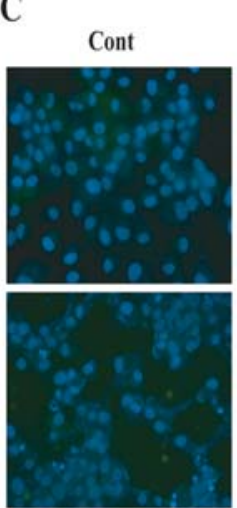

BBR
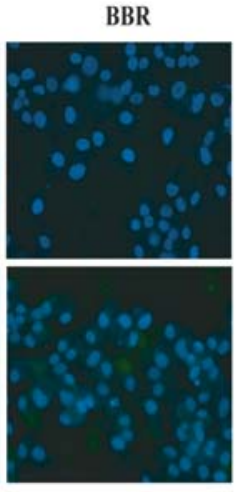

TRAIL

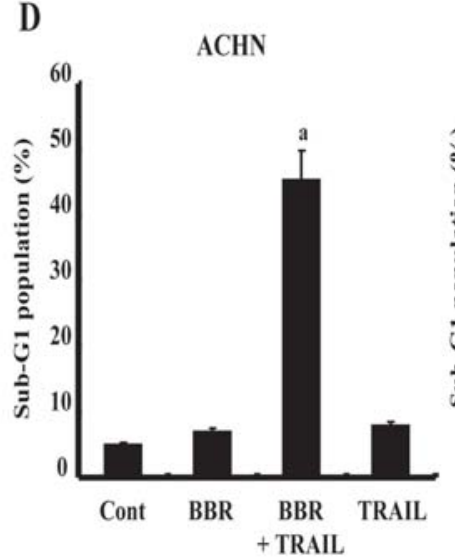

B

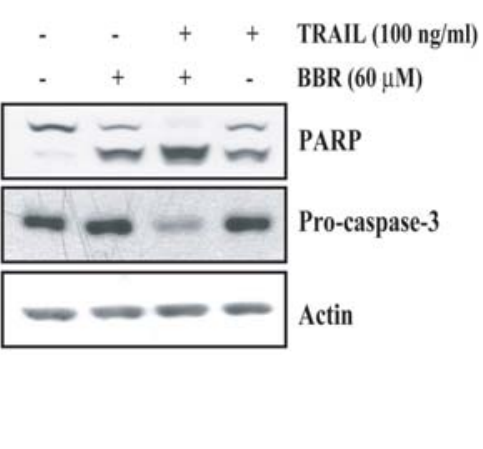

E

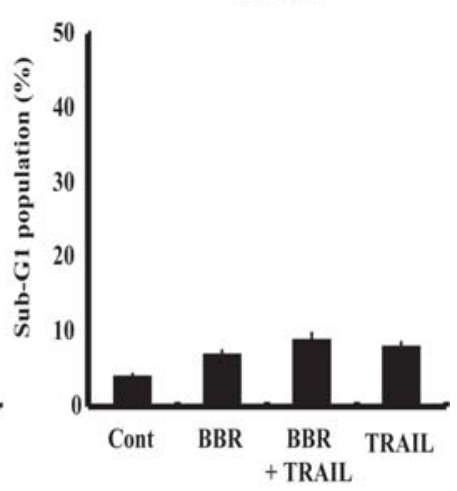

Figure 1. BBR sensitizes renal cancer cells to TRAIL-mediated apoptosis. (A) Caki cells were treated for $24 \mathrm{~h}$ with TRAIL (100 ng/ml) in either the absence or the presence of BBR $(60 \mu \mathrm{M})$. After $24 \mathrm{~h}$ of treatment, apoptosis was analyzed as a sub-G1 fraction by FACS. ${ }^{\mathrm{a}} \mathrm{p}<0.05$ for BBR+TRAIL-treated cells versus control, BBR-, or TRAIL-treated cells by ANOVA. (B) Activation of caspases and cleavage of PARP in BBR-sensitized TRAIL-induced apoptosis. Cells were treated with the indicated concentrations of BBR and TRAIL. Equal amounts of cell lysates (40 $\mu \mathrm{g})$ were subjected to electrophoresis and analyzed by Western blot analysis for PARP, pro-caspase-3, and actin for normalization. (C) After treatment with BBR plus and TRAIL for 24 h, cells were harvested and washed with PBS and exposed to $300 \mathrm{nM}$ 4',6-diamidino-2-phenylindole (DAPI) at room temperature in the dark for 15 min. Samples were observed under a fluorescence microscope. The healthy cell had an oval-shaped cell body, and its chromatin stained dimly and occupied the majority of the cell body. The chromatin of an apoptotic cell was condensed, intensely stained, or shifted to the periphery of the cell body. The morphologies of cells were determined by interference light microscopy (magnification, x200). (D) ACHN cells were treated for $24 \mathrm{~h}$ with TRAIL (100 ng/ml) in either the absence or the presence of BBR $(60 \mu \mathrm{M})$. After $24 \mathrm{~h}$ of treatment, apoptosis was analyzed as a sub-G1 fraction by FACS. ${ }^{\mathrm{a}} \mathrm{p}<0.05$ for BBR+TRAIL-treated cells versus control, BBR-, or TRAIL-treated cells by ANOVA. (E) Mouse tubular normal TCMK-1 cells were treated for $24 \mathrm{~h}$ with TRAIL (100 ng/ml) in either the absence or the presence of BBR $(60 \mu \mathrm{M})$. After $24 \mathrm{~h}$ of treatment, apoptosis was analyzed as a sub-G1 fraction by FACS.

A

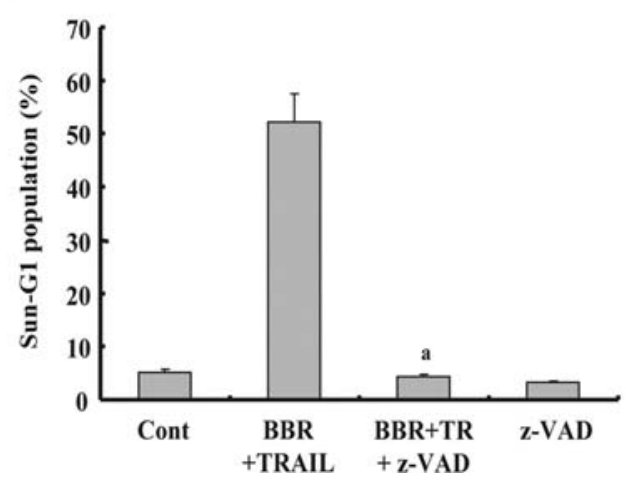

B

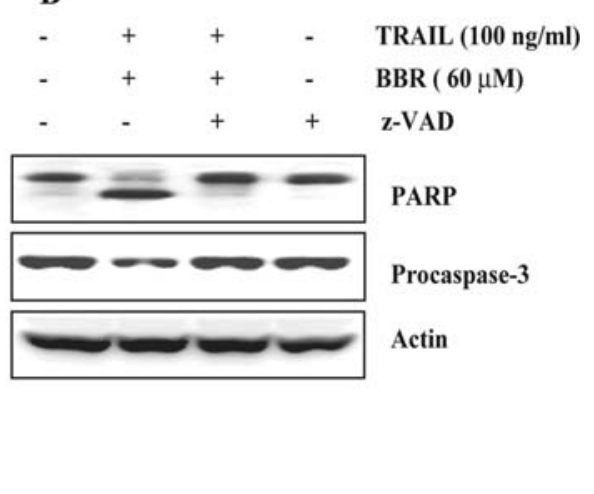

Figure 2. The combined treatment with BBR plus TRAIL induced apoptosis is mediated by caspase-dependent pathway. (A) Effect of z-VAD-fmk on apoptosis induced by BBR plus TRAIL. Caki cells were incubated with $50 \mu \mathrm{M} \mathrm{z}$-VAD-fmk or solvent for $1 \mathrm{~h}$ before treatment with BBR (60 $\mu \mathrm{M})$ and/or TRAIL (100 ng/ml) for $24 \mathrm{~h}$. DNA contents of treated cells were evaluated after propidium iodide staining and apoptosis was measured as a sub-G1 fraction by FACS. Data are mean values obtained from three independent experiments and bars represent standard deviation. ${ }^{\mathrm{a}} \mathrm{p}<0.05$ compared to BBR plus TRAILtreated cells. (B) Effect of z-VAD-fmk on caspase activation in BBR plus TRAIL treated cells. Cells were treated with the indicated concentrations of BBR and TRAIL. Equal amounts of cell lysates $(40 \mu \mathrm{g})$ were subjected to electrophoresis and analyzed by Western blot analysis for procaspase-3 and PARP. The proteolytic cleavage of PARP is indicated by an arrow. A representative study is shown; two additional experiments yielded similar results. 
A

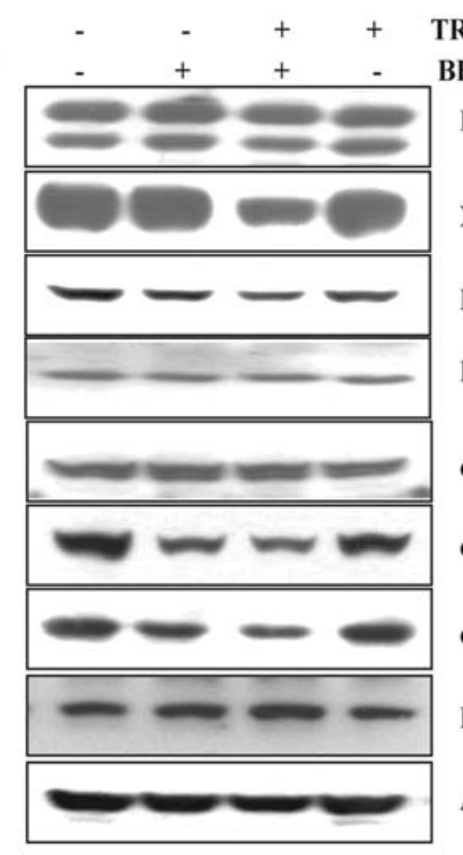

B

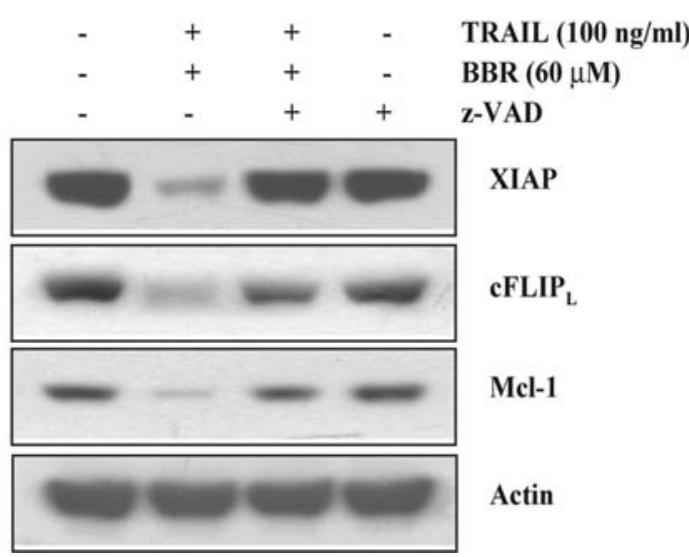

C

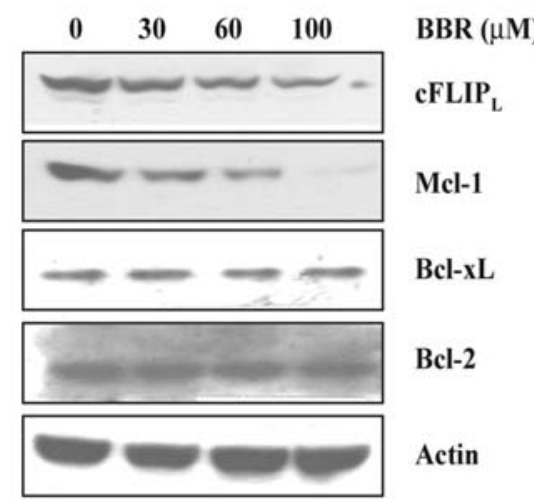

D

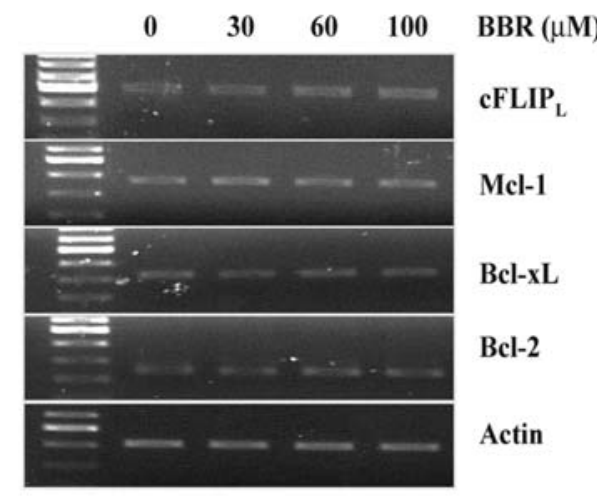

Figure 3. BBR downregulates Mcl-1 and c-FLIP protein levels at post-translational levels. (A) Caki cells were treated with the indicated concentrations of BBR for $24 \mathrm{~h}$ and harvested in lysis buffer. The equal amounts of cell lysates $(40 \mu \mathrm{g})$ were resolved by SDS-PAGE. Western blotting was performed using anti-DR5, anti-XIAP, anti-Mcl-1, anti-Bcl-2, anti-c-IAP1, anti-c-FLIPs, anti-c-FLIP ${ }_{\mathrm{L}}, \mathrm{Bcl}-\mathrm{xL}$ antibodies, or anti-actin antibody to serve as control for the loading of protein level. (B) Effect of z-VAD-fmk on protein expression induced by BBR plus TRAIL. Caki cells were incubated with $50 \mu$ M z-VAD-fmk or solvent for $1 \mathrm{~h}$ before treatment with BBR $(60 \mu \mathrm{M})$ and/or TRAIL $(100 \mathrm{ng} / \mathrm{ml})$ for $24 \mathrm{~h}$. The cells were harvested in lysis buffer, and then equal amounts of cell lysates $(40 \mu \mathrm{g})$ were resolved by SDS-PAGE. Western blotting was performed using anti-XIAP and anti-c-FLIP, anti-Mcl-1 antibodies, or anti-actin antibody to serve as control for the loading of protein level. (C) Caki cells were treated with the indicated times for $24 \mathrm{~h}$ and harvested in lysis buffer and equal amounts of cell lysates $(40 \mu \mathrm{g})$ were resolved by SDS-PAGE. Western blotting was performed using anti-c-FLIP, anti-Mcl-1, anti-Bcl-2 and anti-Bcl-xL antibodies or anti-actin antibody to serve as control for the loading of protein level. (D) Caki cells were treated with the indicated concentrations of BBR for $24 \mathrm{~h}$. Total RNA was isolated and RT-PCR analysis was performed as described in Materials and methods. A representative study is shown; two additional experiments yielded similar results.

Combinational treatment with BBR plus TRAIL-induced apoptosis was mediated by caspase-dependent pathway. We examined whether the activation of caspase pathway plays a critical role in BBR plus TRAIL-induced apoptosis. As shown in Fig. 2A, BBR plus TRAIL-induced apoptosis was completely prevented by pretreatment with a general and potent inhibitor of caspases, z-VAD-fmk, as determined by FACS analysis. These results suggest that the combined treatment with BBR and TRAIL-induced apoptosis was mediated by caspase-dependent apoptosis pathways. We also found that z-VAD-fmk prevented these caspase-related events such as downregulation of procasapse-3 and cleavage of PARP (Fig. 2B).
BBR downregulates Mcl-1 and c-FLIP protein levels at posttranslational level. To investigate the underlying mechanisms involved in the combined treatment with BBR and TRAILinduced apoptosis, we analyzed the expression levels of various apoptosis regulating proteins. While protein levels of XIAP, c-FLIP, and Mcl-1 were remarkably reduced in response to BBR plus TRAIL, the levels of death receptor 5 (DR5), c-IAP1, Bcl-2, and Bcl-xL, did not alter (Fig. 3A). Next, we examined whether the decreased expressions of XIAP, c-FLIP and Mcl-1 proteins were related to caspase activations. Pretreatment with z-VAD-fmk completely recovered XIAP protein to basal level. In contrast, z-VAD-fmk partly blocked BBR plus TRAIL-induced downregulations of 
A

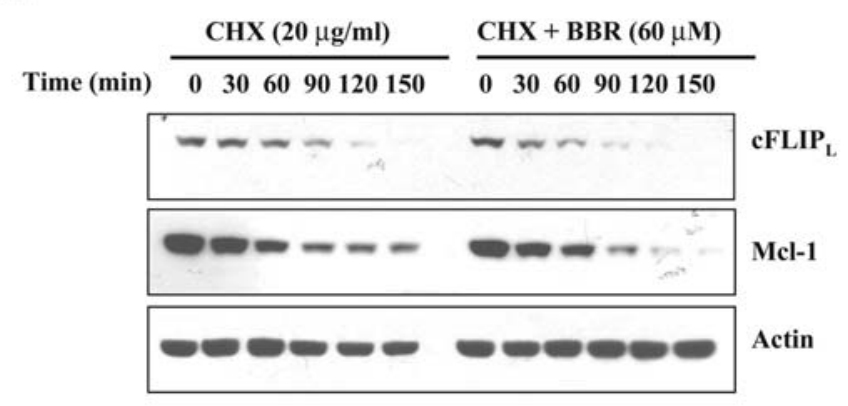

B

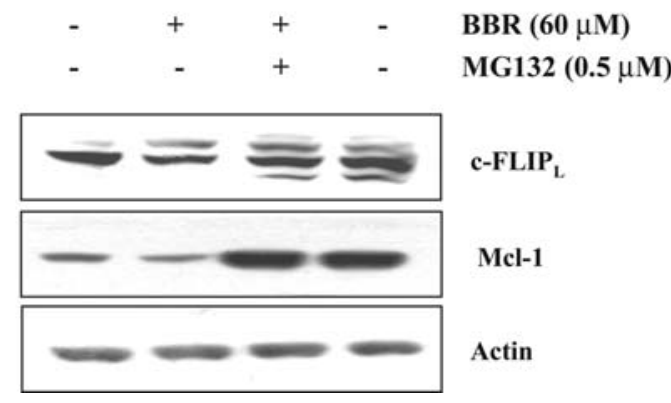

Figure 4. Decreased expressions of c-FLIP and Mcl-1 proteins are caused by the activation of proteasome-dependent pathway in BBR-treated cell. (A) Caki cells were treated with $60 \mu \mathrm{M}$ BBR in the presence or absence of $\mathrm{CHX}$ for the indicated times. Western blotting was performed using anti-c-FLIP $P_{L}$ and anti-Mcl-1 and anti-actin antibody to serve as control for the loading of protein level. (B) Caki cells were treated with $60 \mu \mathrm{M}$ BBR plus CHX in the presence or absence of $0.5 \mu \mathrm{M}$ MG132 for $24 \mathrm{~h}$. Western blotting was performed using anti-c-FLIP $\mathrm{L}_{\mathrm{L}}$ and anti-Mcl-1 antibodies or anti-actin antibody to serve as control for the loading of protein level.

c-FLIP and Mcl-1 proteins, indicating that the decreased cFLIP and Mcl-1 protein levels were partly caused by caspase activation (Fig. 3B). These results suggested the possibility that the decrease of c-FLIP and Mcl-1 proteins were partly mediated by caspase-independent pathways. To ascertain the downregulation of c-FLIP and Mcl-1 by BBR in Caki cells, we carried out time kinetics studies. As shown in Fig. 3C, protein levels of c-FLIP and Mcl-1 were markedly decreased by the indicated concentrations of BBR-treated Caki cells in a dose-dependent manner. To further elucidate the mechanism responsible for the changes in amounts of c-FLIP and Mcl-1 proteins, we determined the levels of c-FLIP and Mcl-1 mRNA by RT-PCR. c-FLIP and Mcl-1 mRNA levels remain constant throughout the BBR treatment at different doses in Caki cells (Fig. 3D). These results suggest the possibility that BBR-mediated degradation of total c-FLIP and Mcl-1 proteins might be regulated by the post-transcriptional levels.

Decreased expressions of $c$-FLIP and Mcl-1 proteins were caused by the activation of proteasome-dependent pathway in BBR-treated cells. To further clarify the underlying mechanisms of the decreased c-FLIP and Mcl-1 protein level in BBR-treated cells, we analyzed the stability of c-FLIP and Mcl-1 proteins. Caki cells were treated with cycloheximide $(\mathrm{CHX})$ plus BBR for different periods of time. We found that the degradations of c-FLIP and Mcl-1 proteins were facili- tated by BBR treatment (Fig. 4A), implying that BBR treatment caused reduction of the c-FLIP and Mcl-1 protein stability. Based on this result, we hypothesized that c-FLIP and Mcl-1 proteins would be degraded by the proteasomedependent pathway. To confirm this hypothesis, we treated the cells with the proteasome inhibitor MG132. Caki cells were treated with BBR $(60 \mu \mathrm{M})$ plus $\mathrm{CHX}$ in the absence or presence of MG132 $(0.5 \mu \mathrm{M})$. As expected, the decreased protein levels of c-FLIP and Mcl-1 in BBR-treated Caki cells were almost recovered to basal levels by MG132 treatment (Fig. 4B).

BBR-stimulated TRAIL-induced apoptosis appears to be dependent on the formation of reactive oxygen species (ROS) via the downregulation of $\mathrm{c}$-FLIP and $\mathrm{Mcl}-1$. Numerous investigations have documented that ROS may play an important role during apoptosis induction $(15,16)$. It has been reported that BBR increases ROS production in various cancer cells $(10,17,18)$. Therefore, we examined whether ROS generation might be involved in BBR plus TRAILinduced apoptosis. $\mathrm{H}_{2}$ DCFDA-based fluorescence microscope detection showed that treatment of Caki cells with BBR increased intracellular ROS levels, which were prevented by anti-oxidant N-acetyl-L-cysteine (NAC) pretreatment (Fig. 5A). We next investigated whether ROS generation is directly associated with BBR plus TRAIL-induced apoptosis. As shown in Fig. 5B, pretreatment with NAC markedly blocked BBR plus TRAIL-induced apoptosis. However, NAC pretreatment recovered the decreased expression levels of c-FLIP by BBR treatment to basal levels, BBR-induced downregulation of Mcl-1 protein was partly blocked by NAC treatment (Fig. 5C). These results suggest the possibility that BBR-stimulated downregulation of c-FLIP protein appears to be dependent on the generation of reactive oxygen species for downregulation of c-FLIP through proteasomal activation.

\section{Discussion}

In the present study, we demonstrate for the first time that treatment of renal cancer cells with BBR in combination with TRAIL synergistically induced apoptosis. c-FLIP and Mcl-1 were downregulated when BBR and TRAIL were administered together, which was caused by facilitating degradation of c-FLIP and Mcl-1 proteins. In addition, we also found that treatment with BBR in combination with TRAIL of Caki cells led to ROS generation, as shown using the fluorescent dyes, $\mathrm{H}_{2}$ DCFDA, which production of ROS by BBR treatment seems to be critical for c-FLIP and Mcl-1 downregulation. Furthermore, combinatory treatment with BBR plus TRAIL synergistically enhanced apoptosis in renal cancer ACHN cells without any cytotoxicity in normal tubular kidney cells.

Several studies demonstrate that TRAIL almost selectively induces apoptosis in cancer cells with no or minimal toxicity on non-neoplastic cells $(1,5)$. However, treatment with TRAIL alone may be insufficient for cancer therapy because some cancers display resistance to TRAIL. In addition, regular consumption of natural compounds such as tea, herbs, gingers, and spices is associated with reduced risk of cancer $(19,20)$. Therefore, much effort to improve the survival of cancer patients also focus on the search for 

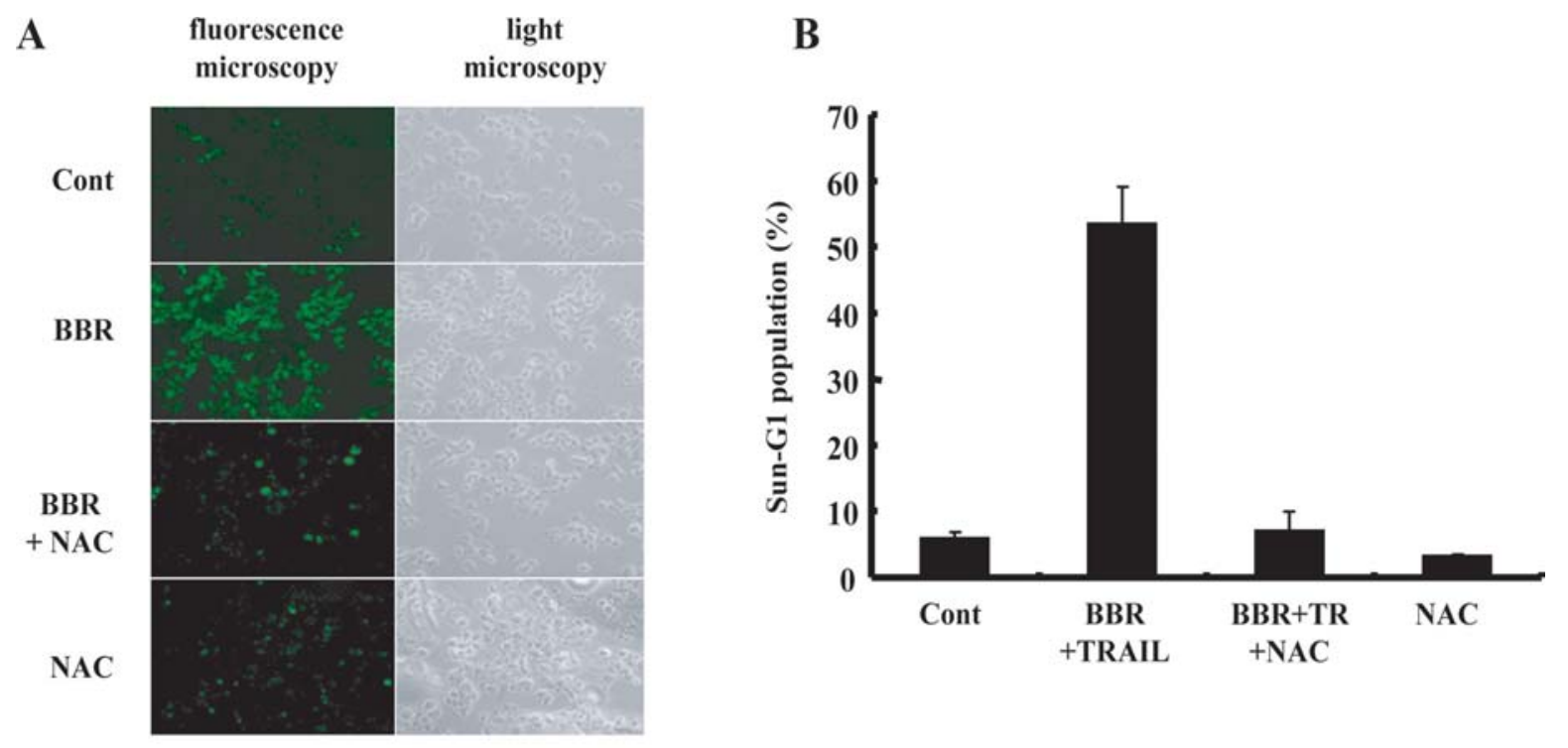

C
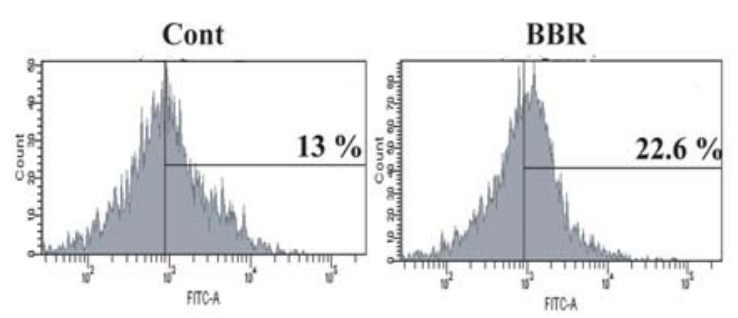

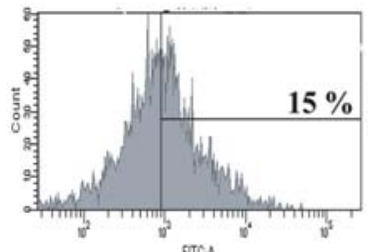

BBR

+ NAC

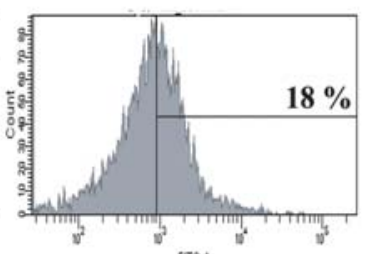

NAC

Figure 5. BBR-stimulated TRAIL-induced apoptosis appears to be dependent on the formation of reactive oxygen species (ROS) via the downregulation of c-FLIP and Mcl-1 proteins. (A) Caki cells were treated with BBR plus TRAIL in the presence or absence of NAC (10 mM). Caki cells were loaded with fluorescence-dye $\mathrm{H}_{2}$ DCFDA and further stimulated with combination of BBR and TRAIL in the presence or absence of NAC (10 mM). $\mathrm{H}_{2}$ DCFDA fluorescence was visualized using a fluorescence microscope (top) or flow cytometry (bottom). (B) Apoptosis was analyzed as a sub-G1 fraction by FACS. ${ }^{a}$ p $<0.05$ for BBR+TRAIL+NAC-treated cells versus BBR+TRAIL-treated cells. (C) Pretreatment with NAC attenuates BBR-induced downregulations of c-FLIP and Mcl-1 proteins. Caki cells were treated with BBR in the indicated concentrations of NAC (2.5-10 mM). Western blotting analysis was performed as above.

natural compounds for treatment of cancer to escape TRAIL resistance or to sensitize cancer cells to TRAIL-induced apoptosis.

Induction of DR5 expression is an important mechanism underlying sensitization of TRAIL-mediated apoptosis (21-25). In this study, we failed to detect any induction of DR5 expression in BBR-treated Caki cells, indicating that DR5 modulation has a restricted role in BBR-induced sensitization of TRAILprompted apoptosis in these cells. Several natural compounds such as triterpenoids, silibinin, and flavopiridol can induce downregulation of c-FLIP and subsequent sensitization to TRAIL-induced apoptosis in cancer cells $(26,27)$. In our present study, treatment with BBR induced downregulation of c-FLIP and the transient expression of c-FLIP partly abrogated induction of apoptosis by combined treatment with BBR and TRAIL (data not shown). These results suggested a critical role of c-FLIP downregulation in mediating the augmentation of TRAIL-induced apoptosis by BBR. It is generally recognized that c-FLIP protein levels can be transcriptionally regulated through the NF- $\mathrm{BB}$ or $\mathrm{c}-\mathrm{Fos}$ pathway $(28,29)$, or by ubiquitin/proteasome-mediated degradation (30-32). In this study, we found that BBR promotes ubiquitin/proteasome-mediated degradation of c-FLIP, leading to downregulation of c-FLIP, but not by transcriptional control.

It was reported that BBR induced the ROS-mediated apoptosis and downregulated the expression of $\mathrm{Bcl}-2$ protein in HepG2 cells, which were prevented by NAC treatment (17). We also found that treatment with BBR induced ROS generation and that pretreatment with NAC prevented BBR plus TRAIL-induced apoptosis. Contrary to this report, we failed to detect any change in Bcl-2 protein levels but found 
marked change of Mcl-1 protein levels by BBR treatment in our system. This controversial result might reflect the differences in the signaling pathways depending on the cellular contexts. Recently, several studies have shown that ROS downregulated c-FLIP or Mcl-1 levels and increased the sensitivity to apoptotic stimuli (33-35). Therefore, we investigated whether downregulation of c-FLIP and Mcl-1 was actually mediated by ROS signaling pathway. In the presence of NAC, the decreased levels of c-FLIP and Mcl-1 caused by BBR were restored. Taken together, BBRstimulated TRAIL-induced apoptosis appears to be dependent on the formation of ROS for downregulation of c-FLIP and $\mathrm{Mcl}-1$ proteins. BBR triggered a proteasomal proteolytic pathway which enhanced protein degradation such as HIF-1ß degradation (36). Parallel to this observation, we observed that BBR triggered c-FLIP and Mcl-1 degradation through a proteasomal proteolytic pathway. However, further study is needed for the mechanistic study to elucidate BBR-induced activation of proteasomal signaling pathway.

In summary, our results provide the first mechanistic evidence that BBR treatment results in ROS-mediated downregulation of c-FLIP and $\mathrm{Mcl}-1$, rendering cancer cells more sensitive to TRAIL. In addition, we suggest that BBR may be a potentially important therapeutic approach for enhancing sensitivity to TRAIL via the inhibition of anti-apoptotic proteins, such as c-FLIP and Mcl-1 in human renal cancer.

\section{Acknowledgements}

This research was supported by the Yeungnam University research grants in 2008 and by the Korea Science and Engineering Foundation (KOSEF) grant funded by the Korea government (MEST) (No. 2010-0007352).

\section{References}

1. Pan G, Ni J, Wei YF, Yu G, Gentz R and Dixit VM: An antagonist decoy receptor and a death domain-containing receptor for TRAIL. Science 277: 815-818, 1997.

2. Sheridan JP, Marsters SA, Pitti RM, et al: Control of TRAILinduced apoptosis by a family of signaling and decoy receptors. Science 277: 818-821, 1997.

3. Fesik SW: Promoting apoptosis as a strategy for cancer drug discovery. Nat Rev Cancer 5: 876-885, 2005.

4. Ozoren N, Fisher MJ, Kim K, et al: Homozygous deletion of the death receptor DR4 gene in a nasopharyngeal cancer cell line is associated with TRAIL resistance. Int J Oncol 16: 917-925, 2000.

5. Wang S and El-Deiry WS: TRAIL and apoptosis induction by TNF-family death receptors. Oncogene 22: 8628-8633, 2003.

6. Ivanov VN, Partridge MA, Johnson GE, Huang SX, Zhou H and Hei TK: Resveratrol sensitizes melanomas to TRAIL through modulation of antiapoptotic gene expression. Exp Cell Res 314: 1163-1176, 2008.

7. Lee TJ, Um HJ, Min do S, Park JW, Choi KS and Kwon TK: Withaferin A sensitizes TRAIL-induced apoptosis through reactive oxygen species-mediated up-regulation of death receptor 5 and down-regulation of c-FLIP. Free Radic Biol Med 46: 1639-1649, 2009.

8. Jung EM, Lim JH, Lee TJ, Park JW, Choi KS and Kwon TK: Curcumin sensitizes tumor necrosis factor-related apoptosisinducing ligand (TRAIL)-induced apoptosis through reactive oxygen species-mediated upregulation of death receptor 5 (DR5). Carcinogenesis 26: 1905-1913, 2005.

9. Eom KS, Hong JM, Youn MJ, So HS, Park R, Kim JM and Kim TY: Berberine induces G1 arrest and apoptosis in human glioblastoma T98G cells through mitochondrial/caspases pathway. Biol Pharm Bull 31: 558-562, 2008.
10. Meeran SM, Katiyar S and Katiyar SK: Berberine-induced apoptosis in human prostate cancer cells is initiated by reactive oxygen species generation. Toxicol Appl Pharmacol 229: 33-43, 2008.

11. Letasiová S, Jantová S, Miko M, Ovádeková R and Horváthová M: Effect of berberine on proliferation, biosynthesis of macromolecules, cell cycle and induction of intercalation with DNA, dsDNA damage and apoptosis in Ehrlich ascites carcinoma cells. J Pharm Pharmacol 58: 263-270, 2006.

12. Kuo CL, Chi CW and Liu TY: The anti-inflammatory potential of berberine in vitro and in vivo. Cancer Lett 203: 127-137, 2004.

13. Yount G, Qian Y, Moore D, et al: Berberine sensitizes human glioma cells, but not normal glial cells, to ionizing radiation in vitro. J Exp Therapeutics Oncol 4: 137-143, 2004.

14. Ho YT, Yang JS, Lu CC, et al: Berberine inhibits human tongue squamous carcinoma cancer tumor growth in a murine xenograft model. Phytomedicine 16: 8878-8890, 2009.

15. Vucicevic L, Misirkic M, Janjetovic K, et al: AMP-activated protein kinase-dependent and -independent mechanisms underlying in vitro antiglioma action of compound C. Biochem Pharmacol 77: 1684-1693, 2009.

16. Sheng-Tanner X, Bump EA and Hedley DW: An oxidative stress-mediated death pathway in irradiated human leukemia cells mapped using multilaser flow cytometry. Radiat Res 150: 636-647, 1998.

17. Hyun MS, Hur JM, Mun YJ, Kim D and Woo WH: BBR induces apoptosis in HepG2 cell through an Akt-ASK1-ROS-p38 MAPKs-linked cascade. J Cell Biochem 109: 329-338, 2010.

18. Ho YT, Lu CC, Yang JS, et al: Berberine induced apoptosis via promoting the expression of caspase- $8,-9$ and -3 , apoptosisinducing factor and endonuclease G in SCC-4 human tongue squamous carcinoma cancer cells. Anticancer Res 29: 4063-4070, 2009.

19. Surh YJ: Cancer chemoprevention with dietary phytochemicals. Nat Rev Cancer 3: 768-780, 2003.

20. Yang CS, Landau JM, Huang MT and Newmark HL: Inhibition of carcinogenesis by dietary polyphenolic compounds. Annu Rev Nutr 21: 381-406, 2001.

21. Moon DO, Kim MO, Choi YH and Kim GY: Butein sensitizes human hepatoma cells to TRAIL-induced apoptosis via extracellular signal-regulated kinase/Sp1-dependent DR5 upregulation and NF-kappaB inactivation. Mol Cancer Ther 9: 1583-1595, 2010.

22. Frese-Schaper M, Schardt JA, Sakai T, Carboni GL, Schmid RA and Frese S: Inhibition of tissue transglutaminase sensitizes TRAIL-resistant lung cancer cells through upregulation of death receptor 5. FEBS Lett 584: 2867-2871, 2010.

23. Kauh J, Fan S, Xia M, Yue P, Yang L, Khuri FR and Sun SY: c-FLIP degradation mediates sensitization of pancreatic cancer cells to TRAIL-induced apoptosis by the histone deacetylase inhibitor LBH589. PLoS One 5: E10376, 2010.

24. Bleumink M, Köhler R, Giaisi M, Proksch P, Krammer PH and Li-Weber M: Rocaglamide breaks TRAIL resistance in HTLV-1associated adult T-cell leukemia/lymphoma by translational suppression of c-FLIP expression. Cell Death Differ (In press).

25. Fan S, Li Y, Yue P, Khuri FR and Sun SY: The eIF4E/eIF4G interaction inhibitor 4EGI-1 augments TRAIL-mediated apoptosis through c-FLIP Down-regulation and DR5 induction independent of inhibition of cap-dependent protein translation. Neoplasia 12: 346-356, 2010.

26. Palacios C, Yerbes R and López-Rivas A: Flavopiridol induces cellular FLICE-inhibitory protein degradation by the proteasome and promotes TRAIL-induced early signaling and apoptosis in breast tumor cells. Cancer Res 66: 8858-8869, 2006.

27. Son YG, Kim EH, Kim JY, et al: Silibinin sensitizes human glioma cells to TRAIL-mediated apoptosis via DR5 up-regulation and down-regulation of c-FLIP and survivin. Cancer Res 67: 8274-8284, 2007

28. Guo F, Sigua C, Tao J, et al: Cotreatment with histone deacetylase inhibitor LAQ824 enhances Apo-2L/tumor necrosis factorrelated apoptosis inducing ligand-induced death inducing signaling complex activity and apoptosis of human acute leukemia cells. Cancer Res 64: 2580-2589, 2004.

29. Watanabe K, Okamoto K and Yonehara S: Sensitization of osteosarcoma cells to death receptor-mediated apoptosis by HDAC inhibitors through downregulation of cellular FLIP. Cell Death Differ 12: 10-18, 2005. 
30. Kim Y, Suh N, Sporn M and Reed JC: An inducible pathway for degradation of FLIP protein sensitizes tumor cells to TRAIL-induced apoptosis. J Biol Chem 2002277: 22320-2239, 2002.

31. Poukkula M, Kaunisto A, Hietakangas V, et al: Rapid turnover of c-FLIPshort is determined by its unique C-terminal tail. J Biol Chem 280: 27345-27355, 2005.

32. Fukazawa T, Fujiwara T, Uno F, et al: Accelerated degradation of cellular FLIP protein through the ubiquitin-proteasome pathway in p53-mediated apoptosis of human cancer cells. Oncogene 20: 5225-5231, 2001.

33. Nitobe J, Yamaguchi S, Okuyama M, et al: Reactive oxygen species regulate FLICE inhibitory protein (FLIP) and susceptibility to Fas-mediated apoptosis in cardiac myocytes. Cardiovasc Res 57: 119-128, 2003.
34. Kanayama A and Miyamoto Y: Apoptosis triggered by phagocytosis-related oxidative stress through FLIPS down-regulation and JNK activation. J Leukoc Biol 82: 1344-1352, 2007.

35. Gao N, Rahmani M, Dent P and Grant S: 2-Methoxyestradiolinduced apoptosis in human leukemia cells proceeds through a reactive oxygen species and Akt-dependent process. Oncogene 24: 3797-3809, 2005

36. Lin S, Tsai SC, Lee CC, Wang BW, Liou JY and Shyu KG: Berberine inhibits HIF-1alpha expression via enhanced proteolysis. Mol Pharmacol 66: 612-619, 2004. 